\title{
Adesão à alimentação escolar e fatores associados em adolescentes de escolas públicas na região Sul do Brasil
}

\author{
Adherence to school food and associated factors among adolescents \\ in public schools in the Southern region of Brazil
}

Josiane Tiborski Cesar (https://orcid.org/0000-0001-6189-7454) ${ }^{1}$

Cesar Augusto Taconeli (https://orcid.org/0000-0002-3485-400X) ${ }^{2}$

Mônica Maria Osório (https://orcid.org/0000-0003-2330-4923) ${ }^{3}$

Suely Teresinha Schmidt (https://orcid.org/0000-0001-6880-0870) ${ }^{1}$

${ }^{1}$ Programa de Pós-

Graduação em Alimentação e Nutrição, Universidade Federal do Paraná (UFPR). Av. Lothário Meissner 632, Jardim Botânico. 80210-170 Curitiba PR Brasil. josi.tice@hotmail.com ${ }^{2}$ Departamento de Estatística, UFPR. Curitiba PR Brasil.

${ }^{3}$ Programa de PósGraduação em Nutrição, Universidade Federal do Pernambuco. Recife PE Brasil.
Abstract The scope of this study was to analyze adherence to school food and associated factors among adolescents from public state schools in the municipality of Lapa, Paraná, in the Southern region of Brazil. The study uses a cross-sectional and analytical design and includes 492 male and female adolescents aged 10 to 19 years from five urban and five rural schools in the municipality. The students underwent anthropometric assessment and answered a questionnaire about school food and alternative food intakes (purchased or home-cooked). Their parents/guardians answered a questionnaire about their demographic and socioeconomic characteristics. An adjusted proportional odds regression model investigated the associations. The effective adherence to school food was 30.1\% in rural schools and $23.7 \%$ in urban schools. Dislike of the food preparation was the main reason for non-adherence. The results showed lesser adherence among adolescents with higher per capita family income who consumed alternative food products four to five times per week. Adolescents who considered school food healthy showed higher adherence. Low adherence to school food indicates the inadequate universality of the program. The factors revealed may help to improve program planning and execution.

Key words Adolescent, School food, Public policies
Resumo Objetivou-se analisar o indice de adesão à alimentação escolar e os fatores associados entre adolescentes matriculados em escolas públicas estaduais do município da Lapa, Paraná, sul do Brasil. De caráter transversal e analítico, incluiu 492 adolescentes de 10 a 19 anos de cinco escolas urbanas e cinco rurais do município. Foi aplicado um questionário sobre o consumo da alimentação escolar e de alimentos competitivos (aqueles comprados ou trazidos de casa) e realizada a avaliação antropométrica nos adolescentes. Os seus pais/responsáveis responderam um questionário demográfico e socioeconômico. Foi utilizado modelo de regressão de "odds" proporcionais ajustado para verificar as associações. $O$ indice de adesão efetiva à alimentação escolar foi de 30,1\% nas escolas rurais e de 23,7\% nas urbanas. Não gostar das preparações servidas foi o principal motivo apontado para a não adesão. Observou-se menor adesão entre os adolescentes que possuíam maior renda familiar per capita e entre os que consumiam alimentos competitivos de 4 a $5 x /$ semana. A adesão foi maior entre aqueles que consideravam a alimentação escolar saudável. Diante do baixo indice de adesão à alimentação escolar, a universalidade do atendimento não está sendo atingida. Os fatores apontados podem auxiliar em melhoras na execução do programa.

Palavras-chave Adolescente, Alimentação escolar, Políticas públicas 


\section{Introdução}

Programas de alimentação escolar estão presentes em 169 países em todo o mundo, beneficiando aproximadamente 368 milhões de estudantes ${ }^{1}$. O fornecimento de uma alimentação adequada e saudável pelas escolas promove a melhoria do estado nutricional, das habilidades cognitivas dos estudantes, contribui para redução da fome e da evasão escolar em áreas onde esse problema persiste. Além disso, muitos programas de alimentação escolar fazem parte do sistema de proteção social dos países, contribuindo para o desenvolvimento local sustentável e da agricultura familiar ${ }^{1}$.

Nesse contexto, o Brasil destaca-se pelo Programa Nacional de Alimentação Escolar (PNAE) que se apresenta como uma das políticas em alimentação e nutrição mais antigas do país. Ao longo de sua história várias reformulações marcantes aconteceram e atualmente sua regulamentação apresenta diretrizes que configuram um modelo de programa exemplar para outros países, sendo uma das estratégias mais importantes de segurança alimentar e nutricional no Brasil e um dos maiores programas de alimentação escolar no mundo ${ }^{2,3}$. O programa, gerenciado pelo Fundo Nacional de Desenvolvimento da Educação (FNDE) e executado pelos estados e municípios, prevê o atendimento universal de forma igualitária a todos os estudantes da rede pública da educação básica ${ }^{4}$.

Ainda que muitos avanços tenham ocorrido, dificuldades são evidenciadas por estudos que apontam, por meio do índice de adesão à alimentação escolar, que apenas um percentual de estudantes consome a alimentação escolar diariamente ${ }^{5-8}$ A Pesquisa Nacional de Saúde do Escolar (PENSE) 2015 apontou que mais da metade dos estudantes $(61,5 \%)$ informou consumir raramente ou nunca a comida ofertada pela escola 9 . Locatelli et al. ${ }^{7}$ trabalhando com dados da mesma pesquisa, porém, do ano de 2012, também evidenciou que apenas $22,8 \%$ dos estudantes consumiam a alimentação escolar três ou mais vezes durante a semana e que esse consumo era maior entre os meninos, entre aqueles que trabalhavam, cujas mães possuíam baixa escolaridade e entre os que tomavam café da manhã e almoçavam com os pais ${ }^{7}$. Outros fatores como idade, estado nutricional dos estudantes, consumo de alimentos competitivos e atividades de educação alimentar e nutricional nas escolas, também são apontados como associados ao consumo da alimentação escolar por outros autores ${ }^{5,6,8}$. Tais estudos, porém, foram conduzidos apenas com estudantes matriculados em escolas da área urbana, fazendo-se necessário também expandir a investigação para outras realidades.

Visto que o ambiente escolar é espaço estratégico de formação de práticas saudáveis de alimentação e nutrição dos estudantes, para que os objetivos do Programa sejam alcançados, é fundamental o consumo adequado da alimentação oferecida nas escolas. O princípio da universalidade do PNAE somente será alcançado de fato, quando todos os estudantes passarem a entender, a reconhecer e a usufruir da alimentação escolar adequada e saudável como seu direito, sendo responsável por essa concretização não só o Estado, mas gestores, membros do programa - responsáveis técnicos, conselhos de alimentação escolar, pais, professores, escolas - e a sociedade civil ${ }^{10}$.

Considerando ainda que o índice de adesão à alimentação escolar apresenta-se como importante ferramenta de monitoramento e investigação da efetividade do PNAE, o objetivo deste estudo foi analisar o índice de adesão à alimentação escolar e os fatores associados entre adolescentes matriculados em escolas públicas estaduais do município da Lapa, Paraná, sul do Brasil.

\section{Métodos}

\section{Desenho do estudo, localização e participantes}

Trata-se de um estudo transversal e analítico realizado com adolescentes, de ambos os sexos, de 10 a 19 anos da rede estadual de ensino do município da Lapa, Paraná, sul do Brasil. O município da Lapa é localizado na região metropolitana de Curitiba, capital do estado do Paraná. O índice de Desenvolvimento Humano (IDH) é alto $(0,706)$ e sua população é de aproximadamente 44.932 habitantes. O município possui uma ampla extensão territorial e um grau de urbanização de 60,6\%. Possui 12 escolas públicas estaduais distribuídas na área urbana e rural ${ }^{11}$.

Para o dimensionamento amostral foi considerado o número de estudantes matriculados nas cinco escolas rurais e nas cinco urbanas de ensino fundamental e médio, período manhã e tarde, existentes no município, no ano de 2015. Nesse ano havia 1.061 estudantes matriculados em escolas da área rural e 2.791 em escolas da área urbana, totalizando 3.852 estudantes. Não fizeram parte da amostra duas das 12 escolas estaduais do município, em virtude de uma apresentar ensi- 
no integral e a outra por destinar-se a adultos. Também foram excluídas as turmas noturnas, de ensino técnico e as de período integral.

Os parâmetros utilizados para o dimensionamento amostral foram: (i) prevalência de adesão à alimentação escolar de 60,0\%, com base em estudo realizado em um município da mesma região do $\mathrm{Brasil}^{8}$, (ii) nível de confiança de 95,0\% e (iii) margem de erro de 0,04 , chegando-se ao total de 501 estudantes. Adicionalmente, foram acrescidos 30,0\% ao total amostral para possíveis perdas e recusas durante a coleta de dados, sendo então 652 estudantes selecionados para a pesquisa. A amostra foi estratificada proporcionalmente à distribuição dos adolescentes em escola e nível de ensino (fundamental e médio). Os estudantes foram selecionados de maneira aleatória a partir da listagem de matrícula das escolas do ano de 2015. No total, 492 adolescentes participaram da pesquisa, o que configurou numa perda amostral efetiva de $1,8 \%$. Os principais motivos para as perdas do estudo foram as faltas no dia da coleta de dados, o esquecimento de trazer o termo de consentimento assinado pelos pais/responsáveis e a recusa do adolescente em participar.

Foi realizado o estudo piloto em uma turma de 35 estudantes, escolhida aleatoriamente de uma escola rural, para testar e ajustar os instrumentos de coleta de dados. Por não ter havido necessidade de reajuste no instrumento da pesquisa, os adolescentes desta escola foram incluídos na amostra.

\section{Características socioeconômicas e demográficas}

Para os pais/responsáveis foi encaminhado para preenchimento um questionário demográfico e socioeconômico e investigadas as seguintes variáveis: área geográfica do domicílio (urbano ou rural), escolaridade materna (sem escolaridade a quatro anos de estudo; de cinco a oito anos de estudo e acima de nove anos de estudo), ocupação dos pais (empregado(a) com carteira assinada, trabalha por conta própria, servidor(a) público/aposentado(a)/pensionista, agricultor(a), do lar/dona de casa, outros), número de moradores no domicílio e a renda familiar mensal. A renda familiar mensal per capita foi categorizada em classes segundo o salário mínimo vigente no Brasil no período da coleta de dados ( $\mathrm{R} \$ 788,00)$ : $<1 / 4$ do salário mínimo; $\geq 1 / 4$ a $1 / 2$ salário mínimo; $\geq 1 / 2$ a um salário mínimo; $\geq$ um salário mínimo.

\section{Questionário sobre alimentação escolar}

O questionário utilizado foi adaptado de Valentim et al. ${ }^{8}$ com a retirada de algumas questões que não eram de interesse do estudo (referentes à estrutura física das escolas, ao preparo e à distribuição das refeições). Esse questionário semiestruturado foi dividido em bloco de questões: (I) para todos os adolescentes; (II) somente para os que não consumiam a alimentação escolar; (III) somente para os que consumiam a alimentação escolar; (IV) somente para quem costumava comprar ou trazer lanches para consumir na escola (alimentos competitivos).

Com a questão "Quantos dias na semana você costuma comer a alimentação oferecida pela escola?" foi obtida a principal variável investigada no estudo e as respostas foram categorizadas segundo os parâmetros utilizados por Sturion et al. ${ }^{5}$ e Valentim et al. ${ }^{8}$ em: não adesão (não consumo); adesão parcial (consumo de um a três vezes na semana) e adesão efetiva (consumo de quatro a cinco vezes na semana). As demais variáveis investigadas foram: frequência semanal do consumo de alimentos competitivos (não consumo, uma a três vezes, quatro a cinco vezes); alimentarse antes de ir à escola (sim ou não); considerar a alimentação escolar saudável (sim ou não). Essas variáveis foram obtidas de questões respondidas por todos os adolescentes.

Os adolescentes que responderam o bloco II também foram questionados sobre o principal motivo para o não consumo da alimentação escolar. Para aqueles que responderam o bloco III, foi questionado qual era o principal motivo para o consumo da alimentação escolar. Por fim, para aqueles que responderam o bloco IV foi questionado qual era o principal motivo para o consumo de alimentos competitivos, quais os locais de compra e quais os alimentos competitivos mais consumidos.

O questionário era autoaplicável, sendo permitido ao adolescente assinalar apenas uma alternativa por questão e todas as questões eram de preenchimento obrigatório.

\section{Medidas antropométricas}

Os dados antropométricos foram obtidos pela aferição do peso em balanças eletrônicas digitais (Marte - capacidade de $180 \mathrm{~kg}$ ) e aferição da estatura em estadiômetro portátil vertical (Alturexata), seguindo procedimentos padronizados $^{12}$. A aferição da estatura foi realizada em duplicata e considerada a média entre as duas 
medidas. No caso de uma variação maior do que $0,5 \mathrm{~cm}$ entre essas duas medidas, novas aferições foram realizadas.

Foi utilizado o software Anthro Plus versão 1.0.4 da World Health Organization (WHO) ${ }^{13}$ para avaliação do estado nutricional, sendo classificado segundo o Índice de Massa Corpórea (IMC). Os resultados foram agrupados em eutrofia (IMC $=\geq$ Escore- $z-2$ a $<$ Escore- $z+1$ ) e excesso de peso $(\geq \text { Escore- } z+1 \text { a }>\text { Escore- } z+3)^{14}$. $\mathrm{O}$ sexo e a data de nascimento dos adolescentes foram obtidos das listas de matrículas das escolas e a idade calculada no software citado acima.

\section{Análise estatística}

A análise dos dados foi feita de forma descritiva e inferencial. As variáveis referentes à opinião dos adolescentes foram analisadas por meio de tabelas de distribuição de frequências com valores absolutos e relativos.

Como possíveis variáveis explicativas à adesão à alimentação escolar foram consideradas: a área geográfica da escola (urbana ou rural), sexo, idade e estado nutricional do adolescente, nível de ensino (fundamental ou médio), se o adolescente se alimentava antes de ir à escola, se considerava a alimentação escolar saudável, qual era a frequência do consumo de alimentos competitivos, a escolaridade da mãe, a renda familiar mensal per capita e a ocupação dos pais.

Primeiramente, foram elaboradas tabelas de distribuição de frequências bivariadas, aplicando-se o teste de qui-quadrado, com o objetivo de verificar possível associação entre as variáveis explicativas com a variável referente à adesão. Aquelas significativas ao nível de $10,0 \%$ na análise bivariada foram consideradas para inclusão no ajuste de modelo de regressão de odds proporcionais ${ }^{15,16}$, considerando como variável resposta o índice de adesão à alimentação escolar. Para essa etapa da análise, foram desconsiderados os adolescentes com informações faltantes, reduzindo o tamanho da amostra para 447 adolescentes.

A suposição de chances proporcionais foi verificada em dois momentos: considerando sua violação para cada uma das covariáveis individuais (por meio do ajuste de modelos de chances proporcionais parciais) e, num segundo momento, considerando sua violação para o conjunto de covariáveis (ajustando-se o modelo de logitos generalizados). Em ambos os casos, não se verificou evidência significativa contrária à suposição de chances proporcionais ao nível de 5,0\%. Dessa forma, a suposição de chances proporcionais não foi rejeitada e o modelo baseado nessa suposição foi adotado. Também foi realizado o teste dos efeitos de todas as interações envolvendo a área geográfica da escola, a fim de verificar se o efeito de alguma covariável diferia em escolas urbanas e rurais. Para todas as interações investigadas, obteve-se $p>0,05$, indicando a não significância desses efeitos. Procedeu-se a análise sem inclusão de efeitos de interação.

Após ajustado o modelo, as variáveis que não apresentaram efeito significativo foram excluídas dessa parte da análise. Adicionalmente, dentre as covariáveis remanescentes, verificou-se, novamente, a necessidade de inclusão do efeito de interação entre as mesmas, o que refletiria efeitos diferenciais de uma covariável segundo os resultados observados de outra. A validação da suposição de chances proporcionais, a avaliação da significância das covariáveis para eventual exclusão do modelo e a análise dos efeitos de interação basearam-se nos resultados de testes de razão de verossimilhanças, adotando-se um nível de significância de 5,0\%.

As análises estatísticas foram realizadas no software estatístico $\mathrm{R}$ versão $3.2 .2^{17}$. O pacote ordinal foi usado para o ajuste do modelo de regressão de odds proporcionais ${ }^{18}$. Os resultados são apresentados através das razões de chances (odds ratios) brutas e ajustadas e correspondentes intervalos de confiança $(95,0 \%)$.

\section{Aspectos éticos}

Este estudo foi realizado de acordo com as orientações definidas na Declaração de Helsinki e todos os procedimentos que envolviam seres humanos foram aprovados pelo Comitê de Ética em Pesquisa do Setor de Ciências da Saúde da Universidade Federal do Paraná. O consentimento informado por escrito foi assinado por um dos pais ou responsáveis legais pelos adolescentes.

\section{Resultados}

Dos 492 adolescentes participantes deste estudo, a maioria era do sexo feminino $(53,3 \%)$ e com idade entre 10 a 14 anos (66,3\%). A prevalência de excesso de peso foi de $31,8 \%$. Em relação às variáveis socioeconômicas, $37,9 \%$ dos adolescentes apresentavam renda familiar per capita entre $1 / 2$ a um salário mínimo e $36,7 \%$ das mães possuíam nove anos de estudo ou mais (Tabela 1 ).

$\mathrm{O}$ índice de adesão à alimentação escolar no município da Lapa, independente da frequência 
Tabela 1. Adesão à alimentação escolar segundo as variáveis demográficas, socioeconômicas e opinião dos adolescentes da rede estadual de ensino da Lapa, PR, 2015

\begin{tabular}{|c|c|c|c|c|c|c|c|c|c|c|}
\hline \multirow[t]{2}{*}{ Variáveis } & \multicolumn{2}{|c|}{ Amostra } & \multicolumn{2}{|c|}{ Não adesão } & \multicolumn{2}{|c|}{$\begin{array}{l}\text { Adesão } \\
\text { parcial }\end{array}$} & \multicolumn{2}{|c|}{$\begin{array}{l}\text { Adesão } \\
\text { efetiva }\end{array}$} & \multirow[t]{2}{*}{$\mathrm{X}^{2}$} & \multirow[t]{2}{*}{ valor-p } \\
\hline & $\mathbf{N}$ & $\%$ & $\mathbf{n}$ & $\%$ & $\mathbf{n}$ & $\%$ & $\mathbf{n}$ & $\%$ & & \\
\hline \multicolumn{11}{|l|}{ Área geográfica da escola } \\
\hline Rural & 146 & 29,7 & 26 & 17,8 & 76 & 52,1 & 44 & 30,1 & \multirow{2}{*}{12,787} & \multirow{2}{*}{$0,002^{\star}$} \\
\hline Urbana & 346 & 70,3 & 117 & 33,8 & 147 & 42,5 & 82 & 23,7 & & \\
\hline \multicolumn{11}{|l|}{ Sexo } \\
\hline Masculino & 230 & 46,7 & 57 & 24,8 & 117 & 50,9 & 56 & 24,3 & \multirow{2}{*}{5,923} & \multirow{2}{*}{$0,052^{\star}$} \\
\hline Feminino & 262 & 53,3 & 86 & 32,8 & 106 & 40,5 & 70 & 26,7 & & \\
\hline \multicolumn{11}{|l|}{ Idade } \\
\hline $10 \mathrm{a} 14$ anos & 326 & 66,3 & 102 & 31,3 & 144 & 44,2 & 80 & 24,5 & \multirow{2}{*}{2,359} & \multirow{2}{*}{0,307} \\
\hline 15 a 19 anos & 166 & 33,7 & 41 & 24,7 & 79 & 47,6 & 46 & 27,7 & & \\
\hline \multicolumn{11}{|l|}{ Período } \\
\hline Manhã & 311 & 63,2 & 83 & 26,7 & 151 & 48,6 & 77 & 24,8 & \multirow{2}{*}{3,826} & \\
\hline Tarde & 181 & 36,8 & 60 & 33,1 & 72 & 39,8 & 49 & 27,1 & & 0,148 \\
\hline Níveis de ensino & & & & & & & & & & \\
\hline Fundamental & 344 & 69,9 & 108 & 31,4 & 153 & 44,5 & 83 & 24,1 & & \\
\hline Médio & 148 & 30,1 & 35 & 23,6 & 70 & 47,3 & 43 & 29,1 & 3,299 & 0,192 \\
\hline Estado Nutricional ( $n$ 463) & & & & & & & & & & \\
\hline Eutrofia & 316 & 68,2 & 90 & 28,5 & 147 & 46,5 & 79 & 25,0 & & \\
\hline Excesso de peso & 147 & 31,8 & 48 & 32,7 & 64 & 43,5 & 35 & 23,8 & 0,839 & 0,657 \\
\hline Renda familiar per capita ( $n$ 467) & & & & & & & & & & \\
\hline$<1 / 4 \mathrm{SM}$ & 66 & 14,1 & 14 & 21,2 & 24 & 36,4 & 28 & 42,4 & & \\
\hline$\geq 1 / 4$ a $1 / 2 \mathrm{SM}$ & 138 & 29,6 & 38 & 27,5 & 72 & 52,2 & 28 & 20,3 & & \\
\hline$\geq 1 / 2$ a $1 S M$ & 177 & 37,9 & 55 & 31,1 & 78 & 44,1 & 44 & 24,9 & 4,623 & $0,023^{*}$ \\
\hline$\geq 1 \mathrm{SM}$ & 86 & 18,4 & 30 & 34,9 & 36 & 41,9 & 20 & 23,3 & & \\
\hline Escolaridade Materna ( $n$ 471) & & & & & & & & & & \\
\hline De zero a quatro anos & 167 & 35,5 & 41 & 24,6 & 78 & 46,7 & 48 & 28,7 & & \\
\hline De cinco a oito anos & 131 & 27,8 & 49 & 37,4 & 56 & 42,7 & 26 & 19,8 & 7,562 & $0,109^{\star}$ \\
\hline Nove anos ou mais & 173 & 36,7 & 46 & 26,6 & 79 & 45,7 & 48 & 27,7 & & \\
\hline Ocupação do pai ( $n$ 459) & & & & & & & & & & \\
\hline Empregado (carteira assinada) & 193 & 42,0 & 61 & 31,6 & 94 & 48,7 & 38 & 19,7 & & \\
\hline Trabalha por conta própria & 99 & 21,6 & 31 & 31,3 & 39 & 39,4 & 29 & 29,3 & & \\
\hline $\begin{array}{l}\text { Servidor Público/Aposentado/ } \\
\text { Pensionista }\end{array}$ & 46 & 10,0 & 16 & 34,8 & 17 & 37,0 & 13 & 28,3 & 9,940 & 0,269 \\
\hline Agricultor & 70 & 15,3 & 13 & 18,6 & 37 & 52,9 & 20 & 28,6 & & \\
\hline Outros & 51 & 11,1 & 15 & 29,4 & 23 & 45,1 & 13 & 25,5 & & \\
\hline Ocupação da mãe ( $n$ 479) & & & & & & & & & & \\
\hline Empregada (carteira assinada) & 119 & 24,8 & 43 & 36,1 & 59 & 49,6 & 17 & 14,3 & & \\
\hline Trabalha por conta própria & 49 & 10,2 & 13 & 26,5 & 27 & 55,1 & 09 & 18,4 & & \\
\hline $\begin{array}{l}\text { Servidora pública/Aposentada/ } \\
\text { Pensionista }\end{array}$ & 69 & 14,4 & 16 & 23,2 & 33 & 47,8 & 20 & 29,0 & 18,892 & $0,042^{\star}$ \\
\hline Agricultora & 51 & 10,6 & 10 & 19,6 & 24 & 47,1 & 17 & 33,3 & & \\
\hline Do lar/dona de casa & 153 & 31,9 & 45 & 29,4 & 61 & 39,9 & 47 & 30,7 & & \\
\hline Outras & 38 & 7,9 & 11 & 28,9 & 14 & 36,8 & 13 & 34,2 & & \\
\hline Alimenta-se antes de ir para escol & & & & & & & & & & \\
\hline Sim & 354 & 72,0 & 93 & 26,3 & 165 & 46,6 & 96 & 27,1 & & $0083 *$ \\
\hline Não & 138 & 28,0 & 50 & 36,2 & 58 & 42,0 & 30 & 21,7 & 4,971 & $0,083^{\circ}$ \\
\hline Considera saudável a alimentação & colar & & & & & & & & & \\
\hline Sim & 423 & 86,0 & 112 & 26,5 & 196 & 46,3 & 115 & 27,2 & 10556 & $0005 x$ \\
\hline Não & 69 & 14,0 & 31 & 44,9 & 27 & 39,1 & 11 & 15,9 & 10,556 & 0,005 \\
\hline Consumo de alimentos competiti & na es & ola & & & & & & & & \\
\hline Não consome & 241 & 49,0 & 56 & 23,2 & 102 & 42,3 & 83 & 34,4 & & \\
\hline Uma a três vezes na semana & 175 & 35,6 & 45 & 25,7 & 94 & 53,7 & 36 & 20,6 & & 0000 * \\
\hline $\begin{array}{l}\text { Quatro a cinco vezes na } \\
\text { semana }\end{array}$ & 76 & 15,4 & 42 & 55,3 & 27 & 35,5 & 07 & 9,2 & 299 & 0,000 \\
\hline
\end{tabular}

SM: Salário Mínimo. ${ }^{*} \mathrm{p}<0,10$. 
do consumo, foi de 70,9\%. Quando analisado por área geográfica, o índice de adesão foi maior nas escolas da área rural $(82,2 \%)$ quando comparada as da área urbana $(66,2 \%)$ (dados não apresentados em tabelas). Observa-se que o índice de adesão efetiva, considerado como o consumo de quatro a cinco vezes na semana, também foi maior entre os adolescentes matriculados em escolas da área rural $(30,1 \%)$.

Com relação à análise bivariada entre as variáveis independentes e as categorias do índice de adesão à alimentação escolar, destaca-se que além da associação significativa com a área geográfica da escola $(p=0,002)$, também se verificou associação com o sexo do adolescente $(\mathrm{p}=0,052)$, a renda familiar per capita $(\mathrm{p}=0,023)$ a escolaridade materna $(\mathrm{p}=0,109)$, a ocupação da mãe ( $\mathrm{p}$ $=0,042$ ), com o fato do adolescente se alimentar antes de ir para escola $(\mathrm{p}=0,083)$ e de considerar a alimentação escolar saudável ( $\mathrm{p}=0,005)$, e com a frequência de consumo de alimentos competitivos na escola $(p=0,000)$ (Tabela 1$)$.

Os resultados referentes ao modelo de regressão de odds proporcionais ajustado para a adesão à alimentação escolar são apresentados na Tabela 2, por meio do odds ratios (OR) e respectivos intervalos de confiança de $95 \%$. Verificou-se que após o ajuste do modelo de regressão de odds proporcionais com as variáveis selecionadas na análise bivariada, duas delas (sexo do adolescente e ocupação da mãe) não apresentaram, conjuntamente, contribuição significativa ao ajuste, sendo excluídas da análise $(\mathrm{p}=0,667)$.

Os adolescentes de escolas urbanas apresentam menor adesão à alimentação escolar em relação aos de escolas rurais, com razoável evidência $(\mathrm{OR}=0,477 ;$ IC95\% 0,277-1,000). Também se observa que a adesão à alimentação escolar entre os adolescentes com renda familiar per capita menor que $1 / 4$ do salário mínimo é sempre superior a dos adolescentes com maior renda familiar per capita. Adolescentes cujas mães possuíam de cinco a oito anos de estudo apresentam menor adesão à alimentação escolar em relação às demais categorias. Entre os adolescentes que consumiam alimentos competitivos de quatro a cinco vezes na semana, a chance de maior adesão é de 23,3\% em relação àqueles que não consumiam esses

Tabela 2. Modelo de regressão de odds proporcionais ajustado para a adesão à alimentação escolar e os fatores associados de adolescentes da rede estadual de ensino da Lapa, PR, 2015

\begin{tabular}{|c|c|c|c|c|c|}
\hline \multirow{2}{*}{ Variáveis } & \multicolumn{2}{|r|}{ Bruto } & \multicolumn{2}{|c|}{ Ajustado } & \multirow[t]{2}{*}{ valor-p } \\
\hline & OR & IC95\% & OR & IC95\% & \\
\hline \multicolumn{6}{|l|}{ Área geográfica da escola } \\
\hline Rural & 1 & - & 1 & - & \\
\hline Urbana & 0,433 & $(0,182-1,024)$ & 0,477 & $(0,277-1,000)$ & 0,050 \\
\hline \multicolumn{6}{|l|}{ Alimenta-se antes de ir para escola } \\
\hline Não & 1 & - & 1 & - & \\
\hline Sim & 1,736 & $(1,158-2,604)$ & 1,623 & $(1,071-2,463)$ & 0,023 \\
\hline \multicolumn{6}{|c|}{ Considera saudável a alimentação escolar } \\
\hline Não & 1 & - & 1 & - & \\
\hline Sim & 2,457 & $(1,426-4,219)$ & 2,364 & $(1,344-4,167)$ & 0,003 \\
\hline \multicolumn{6}{|l|}{ Escolaridade materna } \\
\hline De zero a quatro anos & 1 & - & 1 & - & \\
\hline De cinco a oito anos & 0,655 & $(0,418-1,027)$ & 0,626 & $(0,392-0,997)$ & 0,049 \\
\hline Nove anos ou mais & 1,043 & $(0,678-1,604)$ & 1,348 & $(0,850-2,138)$ & 0,204 \\
\hline \multicolumn{6}{|l|}{ Renda familiar per capita } \\
\hline$<1 / 4 \mathrm{SM}$ & 1 & - & 1 & - & \\
\hline$\geq 1 / 4$ a $1 / 2 \mathrm{SM}$ & 0,509 & $(0,285-0,909)$ & 0,458 & $(0,252-0,832)$ & 0,010 \\
\hline$\geq 1 / 2$ a $1 \mathrm{SM}$ & 0,499 & $(0,281-0,887)$ & 0,431 & $(0,236-0,788)$ & 0,006 \\
\hline$>1 \mathrm{SM}$ & 0,405 & $(0,210-0,781)$ & 0,393 & $(0,196-0,788)$ & 0,008 \\
\hline \multicolumn{6}{|c|}{ Consumo de alimentos competitivos na escola } \\
\hline Não consome & 1 & - & 1 & - & \\
\hline Uma a três vezes na semana & 0,666 & $(0,452-0,983)$ & 0,694 & $(0,467-1,030)$ & 0,070 \\
\hline Quatro a cinco vezes na semana & 0,217 & $(0,122-0,386)$ & 0,233 & $(0,129-0,422)$ & $<0,001$ \\
\hline
\end{tabular}

SM: Salário Mínimo. OR: Odds ratios. IC: Intervalo de confiança. Para o modelo de regressão de odds proporcionais foram considerados apenas os adolescentes com informações para todas as variáveis $(n 447)$. 
alimentos (OR = 0,233, IC95\% 0,129-0,422). Ainda, a chance de maior adesão à alimentação escolar é mais que o dobro entre os adolescentes que consideravam a alimentação escolar saudável e o fato de o adolescente alimentar-se antes de ir a escola aumenta $62,3 \%$ à chance de maior adesão (Tabela 2).

$\mathrm{Na}$ Tabela 3, observa-se que o principal motivo apontado pelos adolescentes para o consumo da alimentação escolar foi sentirem fome no horário do lanche. Dentre os que não consumiam a alimentação escolar, os motivos apontados foram não gostar das preparações servidas, considerar o tempo de espera na fila demorado e o intervalo para realizar a refeição curto. Mais da metade dos adolescentes avaliados afirmaram que consumia outros alimentos durante o período que permanecia na escola e, destes, a maioria o fazia por não gostar da alimentação oferecida no dia. Os alimentos mais consumidos foram salgados fritos e eram comprados na cantina comercial.

\section{Discussão}

Neste estudo o índice de adesão à alimentação escolar esteve associado com a área geográfica da escola, características socioeconômicas, opinião dos adolescentes sobre a alimentação escolar e o consumo de alimentos competitivos. O índice de

Tabela 3. Principais motivos para a não adesão e a adesão à alimentação escolar e o consumo de alimentos competitivos entre os adolescentes da rede estadual de ensino da Lapa, PR, 2015.

\begin{tabular}{|c|c|c|}
\hline & $\mathbf{n}$ & $\%$ \\
\hline \multicolumn{3}{|l|}{ Motivos de adesão à alimentação escolar * (n 349) } \\
\hline Tenho fome na hora do lanche & 169 & 48,4 \\
\hline É saudável & 66 & 18,9 \\
\hline Eu gosto das preparações & 58 & 16,6 \\
\hline Não me alimento antes de ir para a escola & 24 & 6,9 \\
\hline Meus amigos comem & 23 & 6,6 \\
\hline O preço dos alimentos vendidos na cantina é alto & 09 & 2,6 \\
\hline \multicolumn{3}{|l|}{ Motivos de não adesão à alimentação escolar ${ }^{* *}(n$ 143) } \\
\hline Não gosto das preparações & 55 & 38,5 \\
\hline O tempo esperado na fila é demorado & 35 & 24,5 \\
\hline O intervalo para a refeição é curto & 30 & 21,0 \\
\hline Não gosto do horário em que é servida a alimentação escolar & 15 & 10,5 \\
\hline Não há mesas e cadeiras suficientes & 05 & 3,5 \\
\hline A higiene do local é inadequada & 03 & 2,1 \\
\hline \multicolumn{3}{|c|}{ Motivos para o consumo de alimentos competitivos no ambiente escolar ${ }^{\star * \star}(n$ 251) } \\
\hline Não gosto da alimentação oferecida naquele dia & 206 & 82,1 \\
\hline Para complementar a alimentação oferecida pela escola & 29 & 11,5 \\
\hline Porque meus amigos compram & 16 & 6,4 \\
\hline \multicolumn{3}{|l|}{ Local de compra de alimentos competitivos ${ }^{* * *}(n$ 251) } \\
\hline Cantina Comercial da escola & 142 & 56,6 \\
\hline Vendas próximas da escola/supermercados e bares & 94 & 37,4 \\
\hline Traz de casa & 15 & 6,0 \\
\hline \multicolumn{3}{|l|}{ Alimentos competitivos mais consumidos ${ }^{\star \star \star}(n 251)$} \\
\hline Salgados fritos & 100 & 39,8 \\
\hline Salgados assados e sanduíches & 75 & 29,9 \\
\hline Guloseimas (balas, pirulitos, biscoitos recheados, etc.) & 40 & 15,9 \\
\hline Frutas & 17 & 6,8 \\
\hline Salgadinhos tipo chips & 10 & 4,0 \\
\hline Sucos artificiais e refrigerantes & 08 & 3,2 \\
\hline Bolos e tortas & 01 & 0,4 \\
\hline
\end{tabular}


adesão encontrado no município da Lapa foi alto (> 70,0\%) segundo a classificação proposta por Sturion ${ }^{19}$. Ao considerar a área geográfica, entre as escolas da área urbana observou-se um índice médio, oposto às escolas da área rural que apresentaram alto índice de adesão. Porém, diante do caráter universal do programa todos os estudantes deveriam consumir diariamente a alimentação escolar e o índice de adesão efetivo (consumo de quatro a cinco vezes na semana) se apresentou como muito baixo $(<30,0 \%)$ e baixo (de 30,0 a $50,0 \%$ ), na área urbana e rural, respectivamente.

Os índices de adesão efetiva encontrados foram menores do que os apresentados por um estudo de amostragem nacional com crianças e adolescentes de 10 municípios brasileiros $(46,0 \%)^{5}$ e por um estudo em um estado da região sudeste do Brasil $(45,1 \%)^{6}$. Difere também do estudo conduzido no município de Goiás, com crianças e adolescentes de escolas municipais de ensino integral, onde o índice de adesão efetiva para o lanche da manhã foi de $44,0 \%$, para o almoço de $95,0 \%$ e para o lanche da tarde de $78,0 \%{ }^{20}$, valores mais altos devido possivelmente ao fato de passarem o dia todo na escola. Cabe destacar que também são encontradas na literatura outras classificações para o consumo da alimentação escolar, como - não consome regularmente e consome regularmente - utilizado por Locatelli et al. ${ }^{7}$. Ainda que as formas de análises não sejam idênticas, esses resultados apontam que apesar dos avanços ao longo da história do programa ${ }^{3}$, o consumo efetivo da alimentação escolar ainda não é satisfatório.

O princípio da universalidade do atendimento a todos os estudantes da rede pública da educação básica foi incorporado à regulamentação do programa somente com a Lei no 11.947 em 20094, na perspectiva da garantia do Direito Humano à Alimentação Adequada. Nesse sentido, não é suficiente apenas o fornecimento de refeições em todas as escolas, mas um conjunto de procedimentos abrangentes nas esferas da gestão, do controle social e dos beneficiários que possibilitem a concretização efetiva do direito à alimentação escolar ${ }^{10}$, o que é refletido na participação universal dos estudantes no programa. No entanto, o que se observa muitas vezes é que os estudantes não possuem esse entendimento e consideram o PNAE como um programa assistencialista para aqueles mais carentes, que não atende seus anseios e preferências ${ }^{21}$. Ainda assim, mesmo que parte dos estudantes não gostem das preparações servidas, muitos a consomem por ser a única alternativa disponível ${ }^{22}$.
O fato de o estudante não gostar das refeições servidas, pode ser reflexo da inadequação aos seus hábitos e costumes alimentares ${ }^{21}$, da maneira como as refeições são preparadas e servidas ${ }^{20,21}$, e vai além de um cardápio que precisa ser elaborado, de acordo com todas as regulamentações do programa, com um valor per capita repassado pelo FNDE, que varia entre $\mathrm{R} \$ 0,32$ a $\mathrm{R} \$ 1,07^{23}$. Esse valor é insuficiente para o alcance das metas nutricionais do programa cujos cardápios devem fornecer em média 20\% das necessidades nutricionais diárias dos estudantes matriculados em período parcial e $70 \%$ para aqueles que permanecem em período integral ${ }^{24}$. $\mathrm{O}$ valor repassado pelo FNDE deve ser complementado pelos estados e municípios conforme prevê a legislação, no entanto, muitos deles, principalmente em regiões mais pobres, enfrentam dificuldades para complementar os recursos financeiros.

As dificuldades financeiras também resultam no número insuficiente de profissionais. Para toda rede pública estadual de ensino do estado do Paraná, que é de aproximadamente 1,3 milhões de estudantes, o programa estadual conta com apenas três nutricionistas. Essa realidade é encontrada na maioria dos municípios da região Sul do Brasil, onde $71,6 \%$ estão com o quadro técnico inadequado aos padrões numéricos exigidos na legislação, o que interfere no cumprimento de todas as atribuições do nutricionista no PNAE $^{25}$.

O município da Lapa apresenta grande parte da população residente na área rural $^{11}$ e nessa área geográfica o índice de adesão efetiva apresentou-se maior que na urbana. No modelo de regressão de odds proporcionais observou-se que a chance de maior adesão nas escolas urbanas é aproximadamente a metade das escolas rurais. Contudo, as covariáveis analisadas no modelo comportam-se semelhantes em ambas as áreas. Assim, supõe-se que outras situações não investigadas poderiam explicar essa associação, como o tempo de deslocamento até a escola e os hábitos alimentares dos adolescentes.

As escolas rurais analisadas situam-se em áreas distantes e os adolescentes precisam sair muito cedo de casa, se deslocam a pé para ter acesso ao transporte que os levará até a escola. Assim, mesmo que a maioria dos adolescentes residentes na área rural tenha relatado alimentar-se antes de ir à escola (65,8\% - resultado não publicado), o intervalo de tempo até a próxima refeição é longo e, por isso, o principal motivo apontado para o consumo da alimentação escolar foi o fato do adolescente sentir fome na hora do lanche. 
Além disso, também é apontado que na área rural os hábitos alimentares diferenciam-se da área urbana. A Pesquisa Nacional de Orçamentos Familiares (POF-2008/2009) evidencia o maior consumo de alimentos in natura na área rural, enquanto que na urbana destacam-se os produtos processados como biscoitos recheados, sanduíches, salgados fritos e assados e refrigerantes. Os adolescentes que vivem na área rural apresentam maior ingestão de fibras e menor de gordura trans, enquanto os da área urbana consomem em média de $21,0 \%$ a 26,0\% mais açúcares livres do que os da área rural ${ }^{26}$. Portanto, na área rural é maior o consumo de alimentos que configuram uma alimentação adequada e saudável, indo ao encontro da legislação do PNAE, que estabelece o uso de alimentos que respeitem os hábitos alimentares saudáveis, com a elaboração de cardápios seguindo as referências nutricionais ${ }^{24}$. Dessa maneira, possivelmente as preparações dos cardápios oferecidos pelas escolas estejam mais próximas dos hábitos alimentares dessas famílias. No entanto, a avaliação do consumo alimentar desses estudantes não foi objetivo deste estudo, sendo um importante fator a ser investigado em próximas pesquisas com estudantes da área rural, assim como a situação da segurança alimentar dessas famílias. Trivellato et al. ${ }^{27}$ apontaram que é alta a prevalência de Insegurança Alimentar no meio rural brasileiro ${ }^{27}$, sendo este mais um fator que possivelmente reflete no maior consumo da alimentação escolar pelos estudantes da área rural.

A Pesquisa Nacional de Orçamentos Familiares (POF - 2008/2009) aponta que são maiores as prevalências de excesso de peso em adolescentes da área urbana ${ }^{28}$. A alta prevalência de excesso de peso encontrada neste estudo está acima dos percentuais nacionais apresentados pela POF$2008 / 2009^{28}$ e pela literatura internacional ${ }^{29}$, porém corrobora com outros autores ${ }^{30}$ e aponta um quadro preocupante de hábitos alimentares e estilo de vida inadequado dos adolescentes. Hábitos que podem perdurar na vida adulta e que são fatores de risco para as doenças crônicas não transmissíveis ${ }^{31,32}$.

Entre os fatores estudados, merece atenção o fato de o adolescente se alimentar antes de ir à escola estar associado a maior chance de adesão à alimentação escolar. O estudo de Locatteli et al. ${ }^{7}$, que analisou dados da PENSE 2012, evidenciou que os estudantes que tomam café da manhã apresentam maiores chances de consumir a alimentação oferecida na escola. No entanto, ao considerar que um adolescente almoça em casa antes de ir à escola no período da tarde e, que consome mais uma refeição com característica de almoço no intervalo das aulas, duas refeições calóricas estão sendo ofertadas em apenas um período do dia, podendo contribuir para o excesso de peso. Muitas escolas brasileiras costumam apresentar em seus cardápios refeições com características de almoço ou jantar, que não condizem com o horário em que é servida a alimentação escolar (no meio do período da manhã ou da tarde), segundo a opinião dos adolescentes. Essas refeições estão fora do seu contexto habitual e familiar e se tornam estranhas quando oferecidas no intervalo. Para os adolescentes, a alimentação servida deveria estar em sintonia com as atividades que realizam no intervalo, de brincar, correr e $\operatorname{jogar}^{21,33}$. As escolas analisadas neste estudo ofertavam, em sua maioria, refeições com características de almoço ou jantar, como por exemplo: arroz, frango e salada; arroz, feijão, carne bovina, batata-doce e salada; risoto e salada.

No entanto, analisando a situação anteriormente apresentada, dos adolescentes que vivem em áreas rurais distante das escolas, e por isso tem um intervalo longo entre as refeições realizadas em casa e no ambiente escolar, é importante a oferta de refeições completas como um almoço. Para os adolescentes que possuem baixas condições socioeconômicas e, por conseguinte, com dificuldade de acesso e disponibilidade a uma alimentação adequada, essa oferta também é necessária para complementar as refeições realizadas em casa ou até mesmo sendo a principal refeição do dia.

A maior chance de adesão à alimentação escolar em adolescentes de menor renda familiar per capita foi encontrada também por outros autores ${ }^{5,8}$. A importância da condição socioeconômica da família na adesão a alimentação escolar também é confirmada pela maior chance de adesão encontrada entre os adolescentes cujas mães possuem menor escolaridade. A menor escolaridade proporciona menores chances de se ter um emprego com boa remuneração, estando ligada intimamente à renda familiar.

Diante do exposto acima, fica evidente o desafio do PNAE em trabalhar simultaneamente com diversos problemas alimentares e nutricionais enfrentados pelos estudantes brasileiros, como é o caso da dificuldade de acesso a uma alimentação adequada e o excesso de peso, por exemplo. No caso de países mais pobres, a preocupação por vezes é exclusiva e a alimentação escolar pode se apresentar como a única refeição regular e nutritiva que os estudantes têm acesso ${ }^{1}$. 
Por outro lado, nos EUA, o National School Lunch Program (NSLP) atualizou sua regulamentação com normas para melhorar a qualidade da alimentação oferecida em razão da preocupação com altas prevalências de excesso de peso em crianças e adolescentes americanos ${ }^{34}$.

A alimentação escolar no Brasil também é definida como importante ferramenta de Educação Alimentar e Nutricional (EAN). A EAN deve ser pautada em ações estruturantes e em processos de educação permanente em alimentação e nutrição, a fim de formar indivíduos com autonomia de escolha ${ }^{35}$. A EAN pode resultar em estudantes críticos e conscientes quanto às escolhas alimentares e reflete no maior consumo da alimentação escolar. Tal premissa pode ser constatada na chance de maior adesão encontrada, mais que o dobro, para aqueles adolescentes que acreditam que a alimentação escolar é saudável em relação àqueles que não têm essa opinião. No entanto, há muito no que avançar nessa temática quando se observa que na maioria dos municípios da Região Sul do Brasil as ferramentas de EAN mais utilizadas pelos responsáveis técnicos do programa são palestras e atividades lúdicas e recreativas, sendo ainda um desafio à articulação entre vários setores da escola para a inclusão desse tema no currículo escolar ${ }^{25}$.

Além disso, muitas vezes o ambiente escolar não propicia a formação para escolhas alimentares saudáveis, quando apresenta o comércio de alimentos e preparações nutricionalmente inadequadas e a baixo custo nas escolas e proximidades. O comércio de alimentos pelas cantinas comerciais é regulamentado no estado do Paraná por duas legislações ${ }^{36,37}$. No entanto, os alimentos e as preparações mais referidos pelos adolescentes como consumidos fora da alimentação escolar são os de comercialização proibida, por apresentarem altos teores de gordura saturada, gordura trans, açúcar e sal. Verifica-se assim, uma dificuldade no cumprimento da legislação e da fiscalização pelos estados e municípios. Além do fato, de que essa legislação não se aplica ao comércio encontrado nas proximidades das escolas, onde segundo o estudo de Leite et al. ${ }^{38}$ realizado em Santos, situam-se os pontos de vendas que ofertam, em sua maioria, alimentos ultraprocessados.

A disponibilidade de alimentos competitivos nas escolas está relacionada ao menor consumo da alimentação escolar, tanto no Brasil como no EUA, assim como apontado por outros autores ${ }^{5,6,39}$. Um estudo de intervenção realizado em San Francisco (EUA) apontou que a retirada da oferta de alimentos competitivos das escolas aumentou a participação dos estudantes no programa de alimentação escolar americano ${ }^{39}$. Os autores ainda chamam a atenção de que a existência de locais de comércio de alimentos nas escolas pode ser uma prática discriminatória frente aos estudantes de condições socioeconômicas desfavoráveis.

Os achados do presente estudo evidenciam que o índice efetivo de adesão à alimentação escolar é muito baixo nas escolas urbanas e baixo nas escolas rurais, o que leva a crer que os objetivos e as diretrizes do programa não estão sendo alcançados. A universalidade do atendimento não trata somente da oferta da alimentação em todas as escolas, possui uma abrangência mais ampla de fatores que levam ao consumo, à participação efetiva dos estudantes no programa e ao reconhecimento da alimentação escolar como direito de todos, e não apenas de estudantes com condições socioeconômicas desfavoráveis, fator evidenciado neste estudo como relacionado à adesão. Este, e os demais fatores aqui elencados, fornecem subsídios para que gestores possam discutir e avançar na execução do PNAE como política pública promotora do Direito Humano à Alimentação Adequada. 


\section{Colaboradores}

JT Cesar participou do desenho do estudo, coleta de dados, análise e interpretação dos resultados e redação do manuscrito. ST Schmidt e MM Osório contribuíram com a orientação do estudo, interpretação dos resultados e revisão do manuscrito. CA Taconeli contribuiu com as análises estatísticas e revisão do manuscrito.

\section{Agradecimentos}

Os autores agradecem a Secretaria do Estado da Educação do Paraná e a direção das escolas estaduais da Lapa pelo consentimento para realização do estudo, ao Programa de Pós-Graduação em Alimentação e Nutrição da UFPR, e ao apoio financeiro do CNPQ/CAPES - PROCAD/CASADINHO (Conselho Nacional de Desenvolvimento Científico e Tecnológico/Coordenação de Aperfeiçoamento de Pessoal de Nível Superior Programa Nacional de Cooperação Acadêmica).

\section{Referências}

1. World Food Programme (WFP). State of School Feeding Worldwide. Rome: WFP; 2013.

2. Sidaner E, Balaban D, Burlandy L. The Brazilian school feeding programme: an example of an integrated programme in support of food and nutrition security. Public Health Nutr 2013; 16(6):989-994.

3. Sonino R, Torres CL, Schneider S. Reflexive governance for food security: The example of school feeding in Brazil. J Rural Stud 2014; 36:1-12.

4. Brasil. Lei no 11.947, de 16 de junho de 2009. Dispõe sobre o atendimento da alimentação escolar e do Programa Dinheiro Direto na Escola aos alunos da educação básica. Diário Oficial da União 2009; 17 jun.

5. Sturion GL, Silva MV, Ometto AMH, Furtuoso MCO, Pipitone MAP. Fatores condicionantes da adesão dos alunos ao Programa Nacional de Alimentação Escolar no Brasil. Rev Nutr 2005; 18(2):167-181.

6. Silva CAM, Marques LA, Bonomo E, Bezerra OMPA, Corrêa MS, Passos LSF, Souza AA, Barros BF, Souza DMS, Reis JA, Andrade NG. O Programa Nacional de Alimentação Escolar sob a ótica dos alunos da rede estadual de ensino de Minas Gerais, Brasil. Cien Saude Colet 2013; 18(4):963-969.

7. Locatelli NT, Canella DS, Bandoni DH. Fatores associados ao consumo da alimentação escolar por adolescentes no Brasil: resultados da PeNSE 2012. Cad Saude Publica 2017; 33(4):e00183615.

8. Valentim EA, Almeida CCB, Taconeli CA, Osório $\mathrm{MM}, \mathrm{Schmidt} \mathrm{ST}$. Fatores associados à adesão à alimentação escolar por adolescentes de escolas públicas estaduais de Colombo, Paraná, Brasil. Cad Saude Publica 2017; 33(10):e00061016.

9. Instituto Brasileiro de Geografia e Estatística (IBGE). Pesquisa Nacional de Saúde do Escolar 2015 (PENSE). Rio de Janeiro: IBGE; 2016.

10. Siqueira RL, Cotta RMM, Ribeiro RCL, Sperandio N, Priore SL. Análise da incorporação da perspectiva do Direito Humano à Alimentação Adequada no desenho institucional do Programa Nacional de Alimentação Escolar. Cien Saude Colet 2014; 19(1):301-310.

11. Instituto Paranaense de Desenvolvimento Econômico e Social (IPARDES). Caderno Estatístico do Município da Lapa. Curitiba: IPARDES; 2015.

12. Brasil. Ministério da Saúde (MS). Orientações para a coleta e análise de dados antropométricos em serviços de saúde: Norma Técnica do Sistema de Vigilância Alimentar e Nutricional - SISVAN. Brasília: MS; 2011.

13. World Health Organization (WHO). Department of Nutrition for Health and Development. Software for assessing growth and development of the world's children. Geneva: WHO; 2011.

14. World Health Organization (WHO). Multicenter Growth Reference Study Group. WHO child Growth Standards. Length/height-for-age, weight-for-length, weight-for-height and body mass index-for-age. Methods and development. Geneva: WHO; 2006.

15. Agresti A, Kateri M. Categorical data analysis. Berlin: Springer; 2011

16. Abreu MNS, Siqueira AL, Caiaffa WT. Regressão logística ordinal em estudos epidemiológicos. Rev Saude Publica 2009; 43(1):183-194. 
17. R Core Team. R: A language and environment for statistical computing [computer program]. Vienna: R Foundation for Statistical Computing; 2015. [acessado 2016 Jan 15]. Disponível em: https://www.R-project.org/.

18. Christensen RHB. Ordinal - Regression Models for Ordinal Data [computer program]. R package version; 2015. [acessado 2016 Jan 15]. Disponível em: http:// www.cran.r-project.org/package $=$ ordinal/

19. Sturion GL. Programa Nacional de Alimentação Escolar: Avaliação do desempenho em 10 municípios brasileiros [tese]. Campinas: Universidade Estadual de Campinas; 2002.

20. Carvalho NA, Martins KA, Souza LM, Díaz MEP. Feeding in full-time public shcools: Do students adhere and accept? Rev Nutr 2017; 30(3):357-368.

21. Paiva JB, Freitas MCF, Santos LAS. Significados da alimentação escolar segundo alunos atendidos pelo Programa Nacional de Alimentação Escolar. Cien Saude Colet 2016; 21(8):2507-2516.

22. Leme ACB, Phillippi ST, Toassa EC. O que os adolescentes preferem: os alimentos da escola ou os alimentos competitivos? Saude Soc 2013; 22(2):456-467.

23. Brasil. Ministério da Educação (MEC). Resolução no 1, de 8 de fevereiro de 2017. Altera o valor per capita para a oferta da alimentação escolar do Programa de Alimentação Escolar - PNAE. Diário Oficial da União 2017; 9 fev.

24. Brasil. Ministério da Educação (MEC). Fundo Nacional de Desenvolvimento da Educação. Resolução no 26, de 17 de junho de 2013. Dispõe sobre o atendimento da alimentação escolar aos alunos da educação básica no âmbito do Programa Nacional de Alimentação Escolar - PNAE. Diário Oficial da União 2013; 18 jun.

25. Corrêa RS, Rockett FC, Rocha PB, Silva VL, Oliveira VR. Atuação do Nutricionista no Programa Nacional de Alimentação Escolar na Região Sul do Brasil. Cien Saude Colet 2017; 22(2):563-574.

26. Instituto Brasileiro de Geografia e Estatística (IBGE). Pesquisa de Orçamentos Familiares, 2008-2009. Análise do Consumo Alimentar Pessoal no Brasil. Rio de Janeiro: IBGE; 2010.

27. Trivellato PT, Morais DC, Lopes SO, Miguel ES, Franceschini SCC, Priore SE. Insegurança alimentar e nutricional em famílias do meio rural brasileiro: revisão sistemática. Cien Saude Colet 2017; 24(3):865-874.

28. Instituto Brasileiro de Geografia e Estatística (IBGE). Pesquisa de Orçamentos Familiares, 2008-2009. Antropometria e Estado Nutricional de Crianças, Adolescentes e Adultos no Brasil. Rio de Janeiro: IBGE; 2011.

29. Scully M, Wakefield M, Niven P, Chapman K, Crawford D, Pratt IS, Baur LA, Flood V, Morley B. Association between food marketing exposure and adolescents' food choices and eating behaviors. Appetite 2012; 58:1-5.

30. Flores LS, Gaya AR, Petersen RDS, Gaya A. Trends of underweight, overweight, and obesity in Brazilian children and adolescents. J Pediatr 2013; 89(5):456461.
31. Reicks M, Banna J, Cluskey M, Gunther C, Hongu N, Richards R, Topham G, Wong SS. Influence of Parenting Practices on Eating Behaviors of Early Adolescents during Independent Eating Occasions: Implications for Obesity Prevention. Nutrients 2015; 7(10):8783-8801.

32. Schmidt MI, Duncan BB, Azevedo e Silva G, Menezes AM, Monteiro CA, Barreto SM, Chor D, Menezes PR. Chronic non-communicable diseases in Brazil: burden and current challenges. Lancet 2011; 377(9781):1949-1961.

33. Freitas MCS, Minayo MCS, Ramos LB, Fontes GV, Santos LA, Souza EC, Santos AC, Mota SE, Paiva JB, Bernardelli TM, Demétrio F, Menezes I. Escola: lugar de estudar e de comer. Cien Saude Colet 2013; 18(4):979-985

34. US Department of Agriculture. Food and Nutrition Service. Nutrition standards in the National School Lunch and school breakfast programs. Final Rule. $\mathrm{Fe}$ deral Register 2012; 26 jan.

35. Brasil. Ministério do Desenvolvimento Social e Combate à Fome. Marco de referência de educação alimentar e nutricional para as políticas públicas. Brasília: Ministério do Desenvolvimento Social; 2012.

36. Paraná. Lei Estadual no 14.423 , de 2 de junho de 2004. Dispõe que os serviços de lanches nas unidades educacionais públicas e privadas que atendam a educação básica, localizadas no Estado, deverão obedecer a padrões de qualidade nutricional e de vida, indispensáveis à saúde dos alunos. Diário Oficial do Estado 2004; 3 jun.

37. Paraná. Lei Estadual no 14.855 , de 19 de outubro de 2005. Dispõe sobre padrões técnicos de qualidade nutricional, a serem seguidos pelas lanchonetes e similares, instaladas nas escolas de ensino fundamental e médio, particulares e da rede pública. Diário Oficial do Estado 2005; 20 out.

38. Leite FHM, Oliveira MA, Cremm EC, Abreu DSC, Maron LR, Martins PA. Oferta de alimentos processados no entorno das escolas públicas em área urbana. $J$ Pediatr 2012; 88(4):328-334.

39. Bhatia R, Jones P, Reicker Z. Competitive Foods, Discrimination, and Participation in the National School Lunch Program. Am J Public Health 2011; 101(8):1380-1386.

Artigo apresentado em 28/10/2017

Aprovado em 19/07/2018

Versão final apresentada em 21/07/2018 